\title{
Structural, magnetic ordering process and the magnetic excitations in spinel FeMn2O4
}

\author{
Q. ZHANG ${ }^{1}$, W. Tian' ${ }^{2}$, M. Matsuda ${ }^{3}$, A. Christianson ${ }^{4}$, T. Hong ${ }^{5}$, R. Nepal ${ }^{6}$, J. DiTusa ${ }^{7}$, S. Nagler ${ }^{8}$, Ro. \\ $\mathrm{Jin}^{9}$ \\ ${ }^{1}$ No affiliation given, ${ }^{2} \mathrm{ORNL},{ }^{3} \mathrm{ORNL},{ }^{4} \mathrm{ORNL},{ }^{5} \mathrm{ORNL},{ }^{6} \mathrm{ORNL},{ }^{7} \mathrm{ORNL},{ }^{8} \mathrm{ORNL},{ }^{9} \mathrm{ORNL}$ \\ zhangq6@ornl.gov
}

Spinel FeMn2O4 has been reported to exhibit a rough cation distribution of $(\mathrm{Mn} 2+) \mathrm{A}(\mathrm{Mn} 3+\mathrm{Fe} 3+) \mathrm{BO} 4$, with $\mathrm{Mn} 2+$ mainly occupying the tetrahedral (A) sites forming a diamond lattice, and $\mathrm{Fe} 3+$ and $\mathrm{Mn} 3+$ sharing the octahedral (B) sites randomly to form a pyrochlore lattice. Powder neutron diffraction reveals a cubic-tetragonal structural transition at $\sim 595 \mathrm{~K}$ upon cooling due to the Jahn-Teller distortion of MnO6 octahedra. A collinear ferrimagnetic order with antiparallel moments along $\mathrm{c}$ axis between A and B sites is found below $\sim 373 \mathrm{~K}$, and non-collinear ferrimagnetic order induced by the spin canting at B site appears below $\sim 50 \mathrm{~K}$. At $8 \mathrm{~K}$, we observe a spin gap of approximately $5 \mathrm{meV}$ arising from single-ion anisotropy at the orbitally active $\mathrm{Mn} 3+$ site. Spin wave dispersions in both the collinear and non-collinear ferrimagnetic ordered regions have been mapped out via inelastic neutron scattering on large single crystals. We demonstrate that the orbital degree of freedom of B-site Mn3+ ion and its coupling to spin and lattice degrees of freedom play a key role in the structural and magnetic properties in FeMn2O4. 